\title{
Diseño de un portafolio óptimo de suministro eléctrico a partir del modelo de Markowitz: el caso de un usuario en México
}

\author{
Designing an optimal electricity supply portfolio based on the Markowitz model: \\ The case of a user in Mexico
}

\author{
Alfonso Perea González, Osmar Hazael Zavaleta Vazquez* \\ Instituto Tecnológico y de Estudios Superiores de Monterrey, EGADE Business School, México
}

Recibido el el 13 de diciembre de 2017; aceptado el 27 de noviembre de 2018

Disponible en Internet el: 28 de noviembre de 2018

\section{Resumen}

La Reforma Energética de México, promulgada en el año 2013, abrió la posibilidad para que consumidores de energía eléctrica pudieran elegir entre diferentes opciones de suministro. Por lo anterior, el objetivo de este trabajo es validar que el Modelo de Markowitz es útil en la construcción de un portafolio óptimo de suministro de energía eléctrica, a partir de las opciones disponibles en el mercado eléctrico mexicano. Se construyó un caso hipotético, a partir de información real, y los resultados obtenidos en el caso de estudio muestran que el uso de esta metodología permite mitigar los riesgos, a partir de la diversificación de opciones de suministro, tal y como ocurre con la diversificación de alternativas de inversión. Este enfoque constituye una alternativa objetiva para que los usuarios de energía eléctrica planeen y diseñen portafolios óptimos de suministro.

Código JEL: C6, G0, Q4

Palabras clave: Modelo de Markowitz; Portafolio óptimo de suministro eléctrico

\footnotetext{
*Autor para correspondencia

Correo electrónico ozavaleta@itesm.mx (O.H. Zavaleta).

La revisión por pares es responsabilidad de la Universidad Nacional Autónoma de México. 


\begin{abstract}
The Energy Reform in Mexico, which was started in 2013, opened the possibility that electrical energy consumers could choose the supply from any supplier participating in the market. The objective of this work is to validate that Markowitz Model is useful in the construction of an optimal portfolio of electricity supply, from the options available in the Mexican Electricity Market. A hypothetical case, based on real information, was built and the results obtained in the case of study shows that the use of this methodology allows to mitigate risks, from the diversification of supply options, as is the case of the diversification of investment alternatives. This approach constitutes an alternative for consumers of electrical power to plan and design optimal portfolios of electricity supply.
\end{abstract}

JEL codes: $\mathrm{C} 6, \mathrm{G} 0, \mathrm{Q} 4$

Keywords: Markowitz model; Optimal portfolio of energy supply

\title{
Introducción
}

Como resultado de la Reforma Energética [Cámara de Diputados del H. Congreso de la Unión, (2013)] en México, se desprendió la Ley de la Industria Eléctrica [Cámara de Diputados del H. Congreso de la Unión (2014)] con la que se creó un Mercado Eléctrico Mayorista que permite que un usuario pueda acceder al suministro de energía eléctrica, y a sus servicios asociados, de cualquier generador debidamente acreditado.

En paralelo, y durante varios años, coexistirían los Contratos de Interconexión Legados. Esto es, permisos que generadores de energía obtuvieron antes del decreto de la Ley de la Industria Eléctrica, ocurrido en 2014, bajo la modalidad de auto-abasto o cogeneración, esquemas con los que tienen posibilidad de vender energía eléctrica a terceros.

Si bien este escenario abrió oportunidades para que una empresa contrate un servicio de suministro de energía que ofrezca ahorros con respecto a su proveedor actual, también existe el riesgo de que el ahorro no sea el esperado, dada la volatilidad de los precios en periodos de cinco o diez años, característicos de contratos de suministro de energía eléctrica.

La generación de energía eléctrica se realiza a través de fuentes como el gas natural, el agua, el sol, el viento, elementos geotérmicos de la tierra, entre otras. Cada una de estas fuentes ha detonado un proceso particular de generación de energía con su propia estructura de costos, lo que se traduce en una dinámica de precios propia para cada alternativa. El proceso de generación a través de un ciclo combinado, a partir de gas natural, tendría un costo de inversión inicial relativamente bajo, comparado con el gasto operativo que estaría ligado al costo del gas natural, que es el combustible de generación. 
En la generación eólica o en la solar, el costo de la inversión inicial es alto, mientras que los gastos operativos son bajos, lo que se traduce en un costo de generación dependiente del costo del financiamiento de la inversión inicial, el cual sería relativamente estable.

No podemos pasar por alto que, al menos en los primeros años después de la Reforma Energética, la Comisión Federal de Electricidad seguirá siendo un suministrador de diversos usuarios, simplemente porque ofrece energía y potencia de respaldo, así como una garantía de continuidad en el suministro.

Se podría pensar que una solución directa para un usuario sería escoger la opción de menor costo sin embargo, esta alternativa, podría tener un riesgo asociado a la volatilidad en los precios de los combustibles, que es lo que ocurriría, por ejemplo, si se seleccionara el suministro de energía de un proveedor que tiene una planta de generación de ciclo combinado ya que su tarifa está ligada, fundamentalmente, al costo del gas.

En términos de este contexto, el objetivo de este trabajo de investigación es proponer una metodología de análisis, a partir del Modelo de Markowitz (1952), que permita elegir opciones de suministro eléctrico al menor costo y con el menor riesgo.

\section{Revisión de la literatura}

Awerbuch y Berger (2003) emplearon el Modelo de Markowitz (1952) para evaluar proyectos de generación de energía eléctrica en la Unión Europea, incluyendo tanto tecnologías convencionales (carbón, petróleo, gas y nuclear) como renovables (en este caso, eólica).

Por su parte, Awerbuch (2006) analiza el caso de la Unión Europea y añade dos casos de estudio más, uno en los Estados Unidos y otro en México. Concluye que el Modelo de Markowitz es mejor alternativa para la planeación de una mezcla de fuentes de generación de energía eléctrica que un análisis de menor costo, considerando cada opción por separado. El Modelo de Markowitz ayuda a identificar la mezcla que tenga menos riesgo.

DeLaquil, Awerbuch y Stroup (2005) usaron el Modelo de Markowitz en la planeación de la generación de energía eléctrica en el Estado de Virginia en Estados Unidos. Al momento del análisis, la mezcla de fuentes de generación de energía estaba dominada por hidrocarburos, principalmente gas natural, que en esos momentos tenía alta volatilidad y alto costo.

Awerbuch, Jansen, Bursken, y Drennen (2005) utilizan el Modelo de Markowitz para mostrar que el hecho de incluir generación geotérmica y generación renovable en la mezcla de generación eléctrica en la región occidental de los Estados Unidos, cuya tradición de generación es de origen fósil, favorece la reducción de riesgo en el costo de la energía eléctrica.

Woo, Horowitz, Horii y Karimov (2004) proponen el uso del Modelo de Markowitz para la compra de coberturas de energía, para un comercializador de electricidad que quiere mantener costos y riesgos bajos. 
Lesser, Lowengrub y Yang (2007) presentaron a la Comisión de Energía del Estado de California un análisis de mezclas de generación de energía eléctrica para el año 2020. El análisis utilizó un estudio de optimización basado en el Modelo de Markowitz. En este trabajo se concluye que la participación de las tecnologías que no requieren combustibles puede ayudar a reducir el costo y el riesgo del portafolio de generación en California. El uso del Modelo de Markowitz permite analizar la interrelación del costo y riesgo de las alternativas de generación de energía eléctrica.

Huisman, Mahieu y Schlicther (2007) proponen utilizar el Modelo de Markowitz como medida de cobertura en los mercados de energía de un día en adelanto, seleccionando los adecuados contratos en hora punta y en hora no punta, que dan la cobertura en precio y volumen, según la aversión o propensión al riesgo del comprador.

Beltrán (2009) utilizó el Modelo de Markowitz para evaluar el portafolio de generación de energía eléctrica en México. El estudio compara los costos y la varianza de diferentes opciones de generación, entre las que destacan la hidráulica, ciclo combinado, carbón, nuclear, eólica, combustóleo y geotérmica, entre otras. El autor concluye que utilizar métodos de menor costo, para analizar cada opción de generación por separado, puede pasar por alto oportunidades de menor riesgo, lo que no ocurre si se analizan como un portafolio.

Por otro lado, Roques, Hiroux y Saguan (2010) utilizaron el Modelo de Markowitz para analizar el efecto de la diversidad geográfica de los campos de generación eólica ubicados en cinco países europeos (Austria, Dinamarca, Francia, Alemania y España. Se concluyó que el Modelo de Markowitz es una herramienta que puede ayudar a la mejor planeación de un sistema de generación eólica.

Para optimizar la mezcla de generación en Bélgica, Delarue, De Jonghe, Belmans y D’haeseleer (2009) sugieren un modelo en el que se distingue el costo inicial del costo variable de la operación (incluyendo mantenimiento, combustibles y operación en general), a diferencia de otros modelos en los que se usa un costo nivelado. De esta manera se analiza un portafolio de alternativas con fuentes como la nuclear, el carbón, el gas, el petróleo y el viento. Se concluyó que al agregar generación eólica al portafolio su riesgo disminuye.

Roques, Newbery y Nuttall (2007) utilizan técnicas de simulación y el Modelo de Markowitz para evaluar el impacto de los riesgos de los precios de los combustibles, de la electricidad y de emisiones de $\mathrm{CO}_{2}$ para demostrar la utilidad de la diversificación para un inversionista.

Liu y Wu (2010) hicieron un análisis desde la perspectiva de un generador, utilizando el Modelo de Markowitz. El planteamiento sugiere que hay dos maneras de administrar el riesgo en la comercialización de la energía, a través de la diversificación y a través de coberturas. El estudio concluyó que utilizando el Modelo de Markowitz se puede controlar el riesgo, haciendo una asignación apropiada en las diferentes opciones de comercialización, a través del mercado mayorista y mediante contratos bilaterales. La solución óptima sería aquella que 
diera la mejor tasa de rendimiento, para un nivel riesgo dado.

Rodoulis (2010) utiliza el Modelo de Markowitz para analizar la viabilidad de usar gas natural, energía eólica y carbón, junto con petróleo, que hasta ese momento era la principal fuente de generación eléctrica en Chipre. El objetivo fue determinar qué mezcla de portafolio de generación daría la mejor relación costo-riesgo para la isla. Se concluyó que la diversificación planeada de fuentes de generación eléctrica ayuda a reducir el riesgo del portafolio, generado por las fluctuaciones del precio del petróleo.

Yan (2011) emplea el Modelo de Markowitz para desarrollar la propuesta de un portafolio de energía, considerando el costo social como elemento fundamental para consumidores y reguladores, para el caso de los Estados Unidos.

Cucchiella, D’Adamo y Gastaldi (2012) aplicaron el Modelo de Markowitz para analizar diferentes energías renovables en el mercado italiano. Concluyeron que es posible diversificar las fuentes de generación de energía con aquellas de origen renovable, logrando mitigación del riesgo del retorno del portafolio.

Ziegler, Schmitz y Weber (2012) analizaron el mercado interno de Alemania a través del Modelo de Markowitz para encontrar el portafolio óptimo de generación. Para el análisis utilizaron el valor presente neto promedio, a partir de la modelación de los precios de los combustibles.

Lüttgens y Antons (2013) combinan un modelo cualitativo de administración de portafolios de tecnología con el Modelo de Markowitz para evaluar un portafolio de generación de energía, destacando el valor desde una perspectiva holística, no puramente cualitativo y no puramente en la evaluación del riesgo.

En un análisis del caso de Albania, en que el 100\% de la energía eléctrica fue generada por fuentes hidráulicas, Tola (2015) comparó fuentes eólicas y fotovoltaicas en el mismo portafolio. Este comparativo permitió visualizar cómo la diversificación de las fuentes de generación de energía tuvo un efecto en la reducción de riesgo, tal y como ocurre cuando se considera un portafolio de activos financieros.

De igual manera De Llano Paz, Calvo Silvosa y Portos Garcia (2012) utilizaron el Modelo de Markowitz en un caso en España.

Kumar, Mohanta y Reddy (2015) analizaron la información del mercado eléctrico en India, con la finalidad de optimizar un portafolio de alternativas de generación eléctrica que represente el menor precio, dados cierto nivel de riesgo y cierto nivel de emisiones de $\mathrm{CO}_{2}$. Consistente con otros estudios, se concluye que el hecho de considerar diferentes opciones de generación de energía eléctrica reduce el riesgo asociado a las variaciones en los precios de los combustibles para ciertas tecnologías.

En un estudio realizado en el Reino Unido por Adams y Jamasb (2016) se analizó el portafolio de generación que se debería tener para asegurar el suministro de energía eléctrica. 
Utilizando el Modelo de Markowitz, analizaron el portafolio que incluye generación a través de gas (ciclo combinado), nuclear, carbón y eólica, considerando el costo de la inversión, el combustible y el bono de $\mathrm{CO}_{2}$. Se concluyó que una mezcla diversificada puede mitigar el riesgo asociado a la incertidumbre del precio de la electricidad, de los costos de los combustibles y de los costos del bono de carbono para compensar la emisión de $\mathrm{CO}_{2}$. En este estudio se comprobó que considerar un portafolio con fuentes eólicas y fotovoltaicas, como complemento a la generación hidráulica, puede ser adecuado para reducir el riesgo.

Finalmente, Gökgöz y Atmaca (2016) compararon metodologías, basadas en el Modelo de Markowitz, en un análisis para maximizar el retorno y minimizar el riesgo en el mercado eléctrico en Turquía. Se analizó la información de precios, por hora, del día en adelanto y se comparó con el costo de generación de esa misma hora para calcular el retorno. Cada una de las 24 horas del día se considera en el estudio como un activo. Con las metodologías propuestas, se obtienen las horas con más peso en el portafolio óptimo. Los autores incluidos reconocieron que el Modelo de Markowitz fue valioso para fundamentar la toma de decisiones de inversión y para definir regulaciones en un mercado eléctrico.

Esta revisión de la literatura mostró diferentes ámbitos, dentro del sector eléctrico, en los que el Modelo de Markowitz se ha utilizado. En virtud de lo anterior, el objetivo de este trabajo fue utilizar estos conceptos para conformar un portafolio óptimo de suministro de energía eléctrica, desde la perspectiva de un usuario en México. Con este enfoque se pretende que los usuarios, en lugar de tomar decisiones con base en la minimización de costos, únicamente, tomen en cuenta, al mismo tiempo, las consideraciones pertinentes de riesgo con la finalidad de robustecer el proceso de toma de decisiones sobre el suministro de energía eléctrica.

\section{Marco metodológico}

El Modelo de Markowitz (1952) propone, como estrategia de análisis y decisión, una metodología esencialmente estadística y matemática, conocida también como el análisis de media-varianza, para buscar la mejor estrategia de inversión, desde la perspectiva de un inversionista, para balancear los beneficios y atenuar los riesgos.

Markowitz (1952) define que el rendimiento esperado de un portafolio de inversión está dado por:

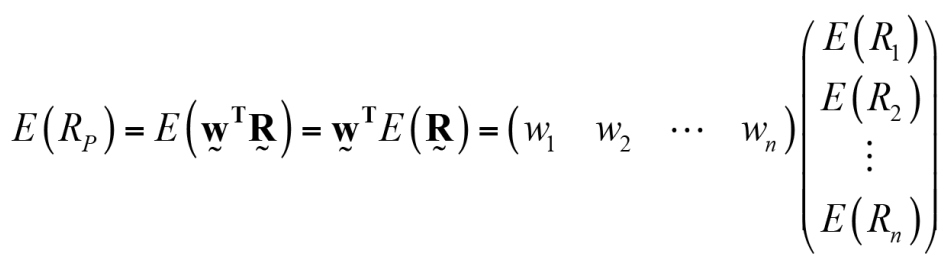


Donde $\underset{\sim}{\mathbf{W}}$ es el vector de ponderaciones, cuyos elementos representan la proporción a invertir en cada uno de los $\mathrm{n}$ instrumentos financieros que integran el portafolio, mientras que $E(\underset{\sim}{\mathbf{R}})$ es el vector de rendimientos esperados, en el que cada elemento representa el rendimiento esperado de cada instrumento del portafolio.

La desviación estándar de los rendimientos del portafolio, que normalmente es una medida de la volatilidad del portafolio, está dada por

$$
\sigma_{P}=\sqrt{\operatorname{Var}\left(R_{P}\right)}=\sqrt{\mathbf{w}^{\mathbf{T}} \operatorname{Var}(\underset{\sim}{\mathbf{R}}) \mathbf{w}}
$$

Donde $\operatorname{Var}(\underset{\sim}{\mathbf{R}})$ es la matriz de varianzas y covarianzas de los rendimientos asociados a los instrumentos que componen el portafolio.

A partir de este contexto, es claro que el objetivo principal del análisis de un portafolio de inversión, a partir de estas ideas, es maximizar su rendimiento, asumiendo cierto nivel de riesgo o volatilidad o bien, minimizar la volatilidad aceptando determinado nivel de rendimiento. Estas ideas se expresan a continuación, como modelos de programación cuadrática:

$\operatorname{Max} E\left(R_{P}\right) \equiv \operatorname{Max}{\underset{\sim}{\mathbf{T}}}^{\mathbf{T}} E(\underset{\sim}{\mathbf{R}})$

Sujeto a:

$\sigma_{P}=\sqrt{\operatorname{Var}\left(R_{P}\right)}=\sqrt{\underset{\sim}{\mathbf{w}^{\mathbf{T}}} \operatorname{Var}(\underset{\sim}{\mathbf{R}}) \underset{\sim}{\mathbf{w}}}=\sigma$

$\stackrel{\sim}{\mathcal{N}}^{T} \underset{\sim}{1}=1$

$\operatorname{Min} \sigma_{P}=\sqrt{\operatorname{Var}\left(R_{P}\right)}=\sqrt{{\underset{\sim}{\mathbf{T}}}^{\mathbf{T}} \operatorname{Var}(\underset{\sim}{\mathbf{R}}) \underset{\sim}{\mathbf{w}}}$

Sujeto a:

$E\left(R_{P}\right)={\underset{\sim}{\mathbf{w}}}^{\mathrm{T}} E(\underset{\sim}{\mathbf{R}})=\mu$

${\underset{\sim}{w}}^{T} \underset{\sim}{1}=1$

Según el Modelo de Markowitz (1952), la diversificación de un portafolio de inversión permite reducir la varianza del rendimiento esperado del portafolio, dada la correlación que hay entre los rendimientos de los instrumentos considerados. Sin embargo, el portafolio que reduce la varianza de los rendimientos no es, por principio, el portafolio que maximiza el rendimiento de la inversión. Estas ideas se pueden comprender de una mejor manera, en términos de la frontera eficiente, la cual muestra la relación entre el rendimiento esperado de un portafolio y su nivel de riesgo, medido a partir de la desviación estándar, como se muestra en la Figura 1. 


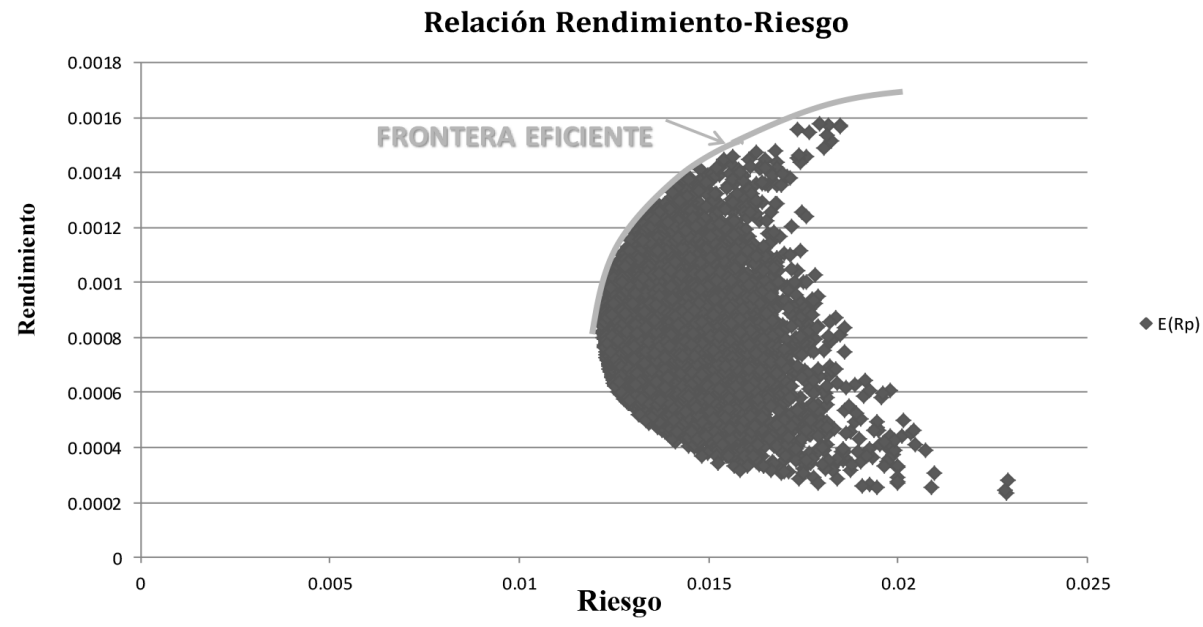

Figura1. Frontera Eficiente y posibles portafolios formados a partir de un conjunto de alternativas de inversión.

Fuente: Elaboración propia

Esta gráfica que ilustra la relación entre Rendimiento y Riesgo fue construida simulando un portafolio de tres activos, a los cuales se les asignaron diferentes ponderaciones. La parte azul de la Figura 1 representa los diferentes portafolios, construidos a partir de diferentes proporciones asignadas a los instrumentos que lo integran. La frontera eficiente corresponde al perímetro superior de la gráfica y representa el conjunto óptimo de portafolios en los que se deberá invertir, dado que se garantiza que en la medida en que se asume más riesgo habrá mayor recompensa en rendimiento. En otras palabras, la frontera eficiente representa el máximo rendimiento, en el conjunto de portafolios, dado cierto nivel de riesgo.

A partir de esta gráfica se puede observar que es posible maximizar el rendimiento del portafolio condicionado a asumir cierto nivel de riesgo.

Una variante en la aplicación de estas ideas es el uso del Modelo de Markowitz en la planeación de un portafolio de generación de energía eléctrica, en un país o en una región. El argumento es que el método usual de planeación de un portafolio de generación analiza el costo de cada fuente de generación por separado. En un análisis así, la fuente de generación seleccionada será siempre la de menor costo. Los costos de las tecnologías tradicionales, a base de carbón y gas, por ejemplo, dependen de los precios de los combustibles y, al ser éstos volátiles, los costos de estas tecnologías se vuelven de alto riesgo.

Al utilizar el Modelo de Markowitz, en lugar de analizar cada fuente de generación por separado, se analiza todo el portafolio de alternativas. Así, se tiene la oportunidad de con- 
siderar las correlaciones entre las diversas fuentes de generación. Las fuentes renovables de generación (como la solar o la eólica) se caracterizan por ser de alto costo de inversión inicial, pero de bajo costo de operación, lo que da como resultado un costo predecible de la energía, a diferencia de las fuentes tradicionales de generación cuyo costo de operación es alto y su costo inicial es, relativamente, bajo, lo que resulta en un costo volátil, dependiente del precio de los combustibles. El Modelo de Markowitz ha permitido incluir, en los portafolios óptimos, las fuentes de generación renovables, debido a que su inclusión permite atenuar el riesgo del costo de la energía del portafolio completo.

\section{Descripción del contexto a estudiar}

En la actualidad, prácticamente todos los usuarios reciben suministro eléctrico por las redes de transmisión y distribución de las nuevas Empresas Productivas del Estado: CFE-Transmisión y CFE-Distribución. La energía que consumen es total o parcialmente generada por alguna de las empresas de CFE-Generación. Al menos en el corto plazo, CFE seguirá suministrando parte del consumo de energía y otros servicios que los usuarios requieran.

Como se ha mencionado, hay generadores que tienen Contratos de Interconexión Legados, que son permisos de generación otorgados por la CFE antes de la emisión de la Ley de la Industria Eléctrica (2014). Estos proveedores tienen permisos de autoabasto o de cogeneración que, total o parcialmente, pueden ofrecer a terceros a través de contratos privados.

Finalmente, un usuario que se registre como Usuario Calificado Participante del Mercado, podrá tener acceso también al mercado mayorista y adquirir energía eléctrica y otros servicios en las subastas del mercado mismo.

Básicamente, hay dos tipos de contratos, pay as you use y take or pay. En un contrato pay as you use, se paga lo que se consume. El precio de la energía eléctrica es acordado y se liquida tanto como se consume.

En un contrato take or pay, la tarifa que se paga es normalmente menor, pero el usuario compra la capacidad acordada y se liquida totalmente, se consuma o no.

Ambos tipos de contrato, con sus respectivas adecuaciones, son los tipos de contrato a los que un usuario podrá acceder, ya sea bajo el régimen de la nueva Ley de Energía Eléctrica o bajo la ley anterior, con generadores con permisos legados.

\section{Portafolio de Suministro a partir del Modelo de Markowitz}

Se utilizó el Modelo de Markowitz (1952), con la finalidad de optimizar un portafolio de alternativas de suministro de energía eléctrica. En el modelo se considera la perspectiva de un usuario que actualmente tiene suministro de energía eléctrica por parte de CFE. 
Al usuario se le presentan opciones de suministro de energía que, en términos generales, se pueden agrupar de la siguiente manera:

1. Continuar su contrato de usuario básico con CFE, en las condiciones actuales.

2. Establecer un contrato de cobertura con un generador que cuenta con un Contrato de Interconexión Legado. En este caso hay generadores que tienen tecnologías a partir de gas natural o tecnologías renovables (como fotovoltaicas o eólicas).

3. Establecer un contrato bilateral bajo la nueva Ley de Industria Eléctrica (2014), el cual podría estar basado en tecnologías a partir de gas natural, tecnologías renovables u otras.

4. Establecer un contrato con un suministrador o con un comercializador, bajo el esquema de Usuario Calificado, a partir de la Ley de la Industria Eléctrica (2014).

5. Participar directamente en el Mercado Eléctrico Mayorista, bajo el esquema de Usuario Calificado Participante del Mercado.

6. Tener una combinación de dos o más de las opciones mencionadas anteriormente.

7. Para el caso de un portafolio con n opciones de suministro de energía eléctrica, el costo esperado por $\mathrm{kW}$-h está dado por:

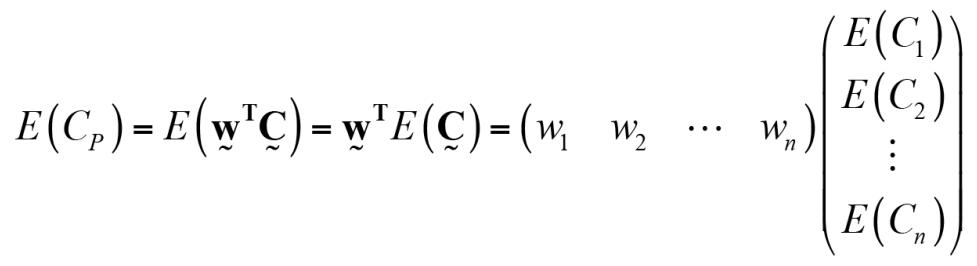

Donde $\underset{\sim}{\mathbf{W}}$ es el vector de ponderaciones, cuyos elementos representan la proporción del suministro total que cada uno de los $n$ suministradores abastecerá, mientras que $E(\underset{\sim}{\mathbf{C}})$ es el vector de costos esperados, en el que cada elemento representa el costo esperado del suministrador correspondiente.

La desviación estándar de los costos del portafolio está descrita por:

$$
\sigma_{P}=\sqrt{\operatorname{Var}\left(C_{P}\right)}=\sqrt{{\underset{\sim}{\mathbf{w}}}^{\mathbf{T}} \operatorname{Var}(\underset{\sim}{\mathbf{C}}) \underset{\sim}{\mathbf{w}}}
$$

Donde $\operatorname{Var}(\underset{\sim}{\mathbf{C}})$ es la matriz de varianzas y covarianzas de los costos de las diferentes alternativas de suministro.

El objetivo es minimizar el costo del proyecto sujeto a un nivel de riesgo apropiado para el usuario, lo que equivale a: 
$\operatorname{Min} E\left(C_{P}\right) \equiv \operatorname{Min} \underset{\sim}{\mathbf{w}} E(\underset{\sim}{\mathbf{C}})$

Sujeto a:

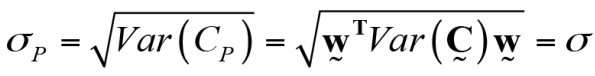

$\stackrel{\sim}{w}^{T} \underset{\sim}{1}=1$

O bien, minimizar el riesgo del portafolio sujeto a un nivel de costo apropiado para el usuario, lo que implica:

$\operatorname{Min} \sigma_{P}=\sqrt{\operatorname{Var}\left(C_{P}\right)}=\sqrt{{\underset{\sim}{\mathbf{w}}}^{\mathrm{T}} \operatorname{Var}(\underset{\sim}{\mathbf{C}}) \underset{\sim}{\mathbf{w}}}$

Sujeto a:

$E\left(C_{P}\right)={\underset{\sim}{\mathbf{w}}}^{\mathbf{T}} E(\underset{\sim}{\mathbf{C}})=c$

$\stackrel{\sim}{T}^{T} \underset{\sim}{1}=1$

\section{Caso de estudio}

Con base en información real, se creó un escenario hipotético con empresas ficticias, en el que una empresa manufacturera enfrenta la toma de decisiones sobre el suministro de energía eléctrica, en un contexto actual en México, a partir de la Reforma Energética.

La empresa Manufacturas de Occidente tiene sus oficinas centrales en Estados Unidos de América. Su operación en México incluye varios puntos de carga ubicados en varios Estados de la República Mexicana.

En 2016, la operación en estos puntos de carga tenía un suministro de energía eléctrica con una demanda contratada de 3,500 kW en tarifa HM con la Comisión Federal de Electricidad. Tenía un consumo anual de 12,400 MW-h, con una tarifa monómica de \$1.34 MXN por kW-h.

En la búsqueda de nuevas ofertas de suministro eléctrico que puedan ofrecer un mejor precio para 2017 y los siguientes años, Manufacturas de Occidente recibió diferentes propuestas. Ahora, debe decidir cuál de las siguientes opciones sería la más conveniente:

1. Continuar su contrato con CFE al $100 \%$ de su capacidad. Si esta no fuera la decisión, de cualquier modo, se requeriría continuar con CFE, al menos con un mínimo consumo, por la necesidad de contar con el respaldo de suministro de energía eléctrica. Según los contratos con CFE en tarifa HM, el pago mínimo mensual resulta de aplicar el cargo por kilowatt de demanda facturable, al $10 \%$ de la demanda contratada. Cabe mencionar que la práctica común es compartir con CFE y otro proveedor el suministro de energía. Dado que la facturación del servicio de energía eléctrica está compuesta 
principalmente de energía y demanda (otros servicios son menores) una posibilidad es que el otro proveedor atienda el consumo completo de la energía y sólo parte de la demanda facturable, dejando el resto de la demanda facturable a CFE, cumpliendo con el requisito del $10 \%$ de la demanda contratada.

2. Establecer un contrato con Universal Energy, que ofrece una tarifa referenciada a la tarifa HM, menos un $16 \%$ de descuento, como tarifa monómica, con un piso de $\$ 1.25$ MXN por kWh, indexada al INPC. El contrato operaría bajo la modalidad de take or pay, por lo que la energía no consumida también se pagaría. Dado que Universal Energy tiene capacidad de generación limpia, a través de un contrato legado, tiene la oportunidad de bancar energía, es decir, generarla en un momento dado, entregarla a la red y, utilizarla en un momento futuro, cuando fuese requerida.

3. Nueva Era, que obtiene su energía de un generador con contrato de interconexión legado a partir de generación eólica, ofrece una tarifa de \$70 USD por MWh, indexada con el USCPI. Éste es un esquema pay as you use.

4. Generador Potosino, que ofrece un contrato pay as you use con una tarifa referenciada a la tarifa HM, con un descuento ponderado de 6\%. El descuento específico por periodo es de $2 \%$ en periodo base, $3.5 \%$ en periodo intermedio y $15 \%$ en periodo punta. Además, un descuento de $8 \%$ en la tarifa de demanda facturable. La fuente de suministro de generación está compuesta de ciclos combinados, cogeneraciones y generación eólica.

5. Establecer un contrato con Comercializadora Buendía, que ofrece un suministro de cogeneración eficiente que contempla descuentos variables, con un máximo del 12\% respecto a la tarifa HM, en modalidad take or pay, a una tarifa monómica por costo de generación, considerando costos de operación y mantenimiento, de 3 centavos de USD/kWh, con un aumento indexado al USCPI, más un costo de porteo de $0.04 \mathrm{MXN} /$ kWh, además del costo del gas natural a partir del precio del gas en México con una eficiencia de 7,200 Btu/kWh. Dado que la generación de Comercializadora Buendía es cogeneración eficiente (generación limpia), tiene la oportunidad de banqueo, con lo que se simplifica el hecho de asegurar que se entregue el $100 \%$ de la energía.

Primeramente, se estimaron los costos promedio esperados para cada una de las opciones. Con la finalidad de estimar medias, varianzas y covarianzas se consideraron datos mensuales, de enero de 2011 a octubre de 2016. Para el análisis, se hizo un cálculo independiente para cada punto de carga y luego se ponderaron las tarifas estimadas por punto de carga para obtener una tarifa final para Manufacturas de Occidente. Para la construcción de la matriz de costos se siguió la siguiente metodología, para cada una de las opciones de suministro: 
1. CFE, cuya tarifa está compuesta por cuatro factores, tres tarifas por energía en periodos punta, intermedia y base, y por demanda facturable (potencia). Como un primer esfuerzo, y con la finalidad de simplificar el análisis, se consideró que todos los meses tuvieron el mismo perfil de consumo, lo cual implicaría que la operación de la empresa era estable a lo largo del año. Dadas las tarifas HM históricas de CFE y con la demanda actual, se construyó una matriz de costos históricos por kW-h con CFE. Finalmente, se deflactaron los costos históricos utilizando el INPC.

2. Universal Energy, con una tarifa referenciada a tarifa HM con descuento de $16 \%$ y un piso de $\$ 1.25$ por $\mathrm{kWh}$ en tarifa monómica. Como no dispusimos de datos históricos, se estimaron directamente de los costos históricos de CFE, deflactados, ajustándolos $16 \%$ abajo y, comparándola con la tarifa piso de $\$ 1.25$ por $\mathrm{kWh}$. Se consideró el $100 \%$ de la energía suministrada por Universal Energy, así como la demanda facturable suministrada. El exceso de demanda facturable es calculado y cubierto por CFE.

3. Nueva Era, con una tarifa en dólares convertida a pesos, utilizando la paridad histórica peso-dólar, deflactada en base al INPC. Se consideró el 100\% de la energía suministrada por Nueva Era, así como la demanda facturable suministrada. El exceso de demanda facturable es calculado y cubierto por CFE.

4. Para Generador Potosino, con una tarifa por periodo y por demanda facturable, se estimó la mezcla de consumo por punto de carga según datos históricos para definir una tarifa monómica por periodo. El exceso de demanda facturable es calculado y cubierto por CFE.

5. Para Comercializadora Buendía, con una tarifa de energía basada en el costo de generación, se construyó la tarifa por periodo, utilizando los costos históricos del gas natural. Se comparó con una tarifa de energía ponderada con $10 \%$ de descuento a la tarifa HM. Se estimó finalmente la tarifa monómica de Comercializadora Buendía. El exceso de demanda facturable es calculado y cubierto por CFE.

6. Una vez construida una tabla de tarifas estimadas para cada opción y para cada punto de carga, de enero de 2011 a octubre de 2016, se ponderó la tarifa con base a la energía consumida en cada punto de carga, para condensarla en una tabla de tarifas monómicas ponderadas (costos comparables), la cual se muestra en la Tabla 1 del Apéndice 1.

A partir de una gráfica a través del tiempo de los costos de las diferentes alternativas, se puede ver que la propuesta en dólares de Nueva Era tiene un comportamiento diferente al de las otras opciones que están referenciadas a la tarifa HM de CFE. Se puede observar que esta tarifa, en dólares, tiende a aumentar en los últimos meses, como se puede ver en la Figura 2. 


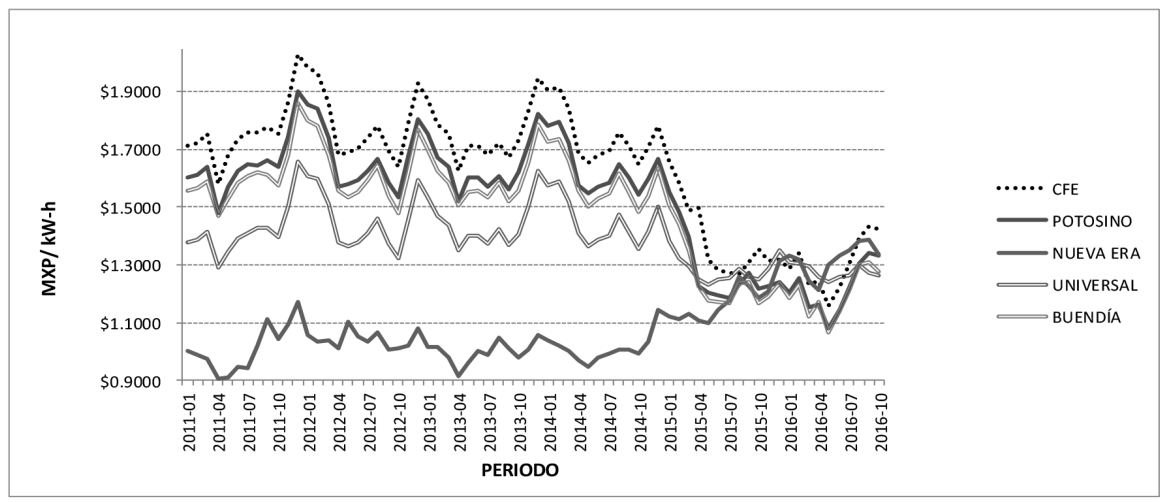

Figura 2. Gráfica de Costos de Opciones de Suministro.

Fuente: Elaboración propia con datos de CFE, Banco de México y la Reserva Federal de los Estados Unidos.

Las medias y desviaciones estándar calculadas de la matriz de costos se incluyen en la Tabla 2 .

Tabla 2

Medias y Desviaciones Estándar de Costos de Energía Eléctrica

\begin{tabular}{|c|c|c|c|c|c|}
\hline & CFE & POTOSINO & NUEVA ERA & UNIVERSAL & BUENDÍA \\
\hline & Tarifa CFE & CFE - $6 \%$ & $\begin{array}{c}70 \text { USD / } \\
\text { MW-h }\end{array}$ & $\begin{array}{c}\text { CFE }-16 \% \text { Cl } \\
\text { PISO }\end{array}$ & $\begin{array}{c}\text { COSTO DE } \\
\text { GENERACIÓN }\end{array}$ \\
\hline MEDIA & 1.63342 & 1.52597 & $\$ 1.08618$ & 1.39108 & $\$ 1.48821$ \\
\hline$\overline{\text { DESV. EST }}$ & 0.22084 & 0.20922 & $\$ 0.12611$ & 0.10541 & $\$ 0.19781$ \\
\hline
\end{tabular}

Fuente: Elaboración propia con datos de CFE, Banco de México y la Reserva Federal de los Estados Unidos.

La matriz de varianzas y covarianzas se muestra en la Tabla 3.

Tabla 3

Varianzas y Covarianzas de Costos de Energía Eléctrica

\begin{tabular}{l|c|c|c|c|c}
\cline { 2 - 6 } & Tarifa CFE & CFE - 6\% & $\begin{array}{c}\text { 70 USD / } \\
\text { MW-h }\end{array}$ & $\begin{array}{c}\text { CFE - 16\% C/ } \\
\text { PISO }\end{array}$ & $\begin{array}{c}\text { BUENDÍA } \\
\text { GENERACIÓN }\end{array}$ \\
\hline Tarifa CFE & 0.048769 & 0.045256 & -0.019229 & 0.020691 & 0.042700 \\
\hline CFE - 6\% & 0.045256 & 0.043773 & -0.017865 & 0.019773 & 0.040688 \\
\hline $\begin{array}{l}\text { CFE - 16\% C/ } \\
\text { PISO }\end{array}$ & 0.020691 & 0.019773 & -0.005882 & 0.011111 & 0.018781 \\
\hline $\begin{array}{l}\text { COSTO DE } \\
\text { GENERACIÓN }\end{array}$ & 0.042700 & 0.040688 & -0.016776 & 0.018781 & 0.039127
\end{tabular}

Fuente: Elaboración propia con datos de CFE, Banco de México y la Reserva Federal de los Estados Unidos. 
La Figura 3 muestra la gráfica del Costo Esperado, $E\left(C_{P}\right)$, en función de la Volatilidad de los Costos, $\sigma\left(C_{P}\right)$, la cual se construyó simulando diferentes ponderaciones a las diferentes opciones de suministro que conforman el portafolio.

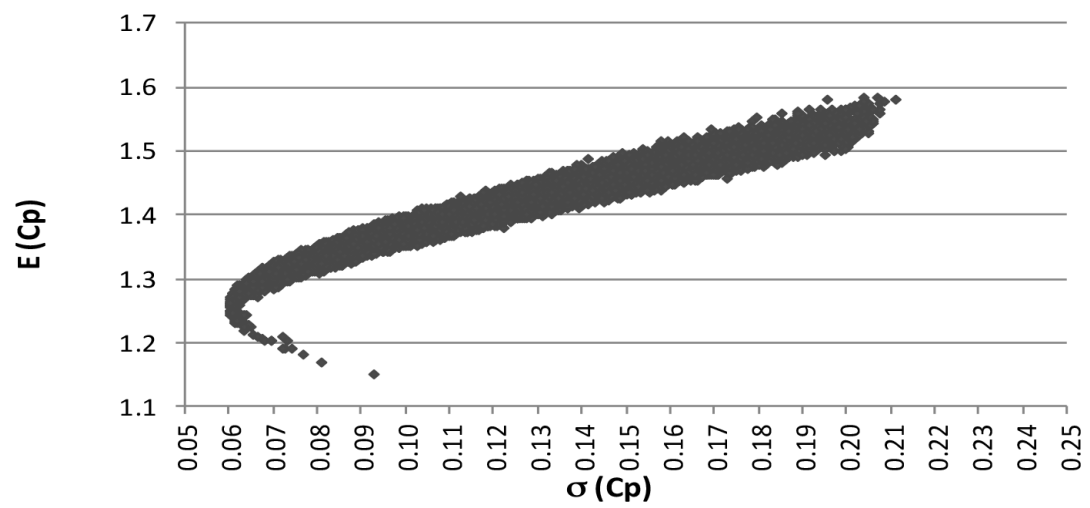

Figura 3. Posibles portafolios de suministro eléctrico

Fuente: Elaboración propia con datos de CFE, Banco de México y la Reserva Federal de los Estados Unidos.

Además de un precio bajo por kW-h de energía eléctrica, Manufacturas de Occidente también busca disminuir el riesgo. Desea asegurar un precio razonablemente estable que le permita hacer planeaciones de mediano y largo plazo de manera confiable, pero está dispuesta a asumir un riesgo moderado que le permita obtener un mejor precio que el portafolio de mínima varianza. Por lo anterior, un nivel de riesgo bajo es el primer cuartil. La metodología empleada no cambiaría si la empresa quisiera asumir mayor o menor riesgo.

La Figura 3 permite identificar que la desviación estándar de los costos de los posibles portafolios varía de 0.05 a 0.21 pesos. La desviación estándar del primer cuartil es de 0.0900 (una varianza de 0.0081 ).

El planteamiento matemático del problema es:

$$
\operatorname{MinE}\left[w_{1} C_{1}+w_{2} C_{2}+w_{3} C_{3}+w_{4} C_{4}+w_{5} C_{5}\right] \equiv \operatorname{Min} \underset{\sim}{\underset{\sim}{T}} E(\underset{\sim}{C})
$$

Sujeta a $\quad \underset{\sim}{\mathbf{w}}{ }_{\sim}^{\mathrm{T}}=1, \sqrt{\operatorname{Var}\left(C_{p}\right)}=\sqrt{\underset{\sim}{\mathbf{W}} \operatorname{Var}(\underset{\sim}{\mathbf{C}}) \underset{\sim}{\mathbf{w}}} \leq 0.09$

$$
{\underset{\sim}{~}}^{\mathrm{T}} \operatorname{Var}(\underset{\sim}{\mathbf{C}}) \underset{\sim}{\mathbf{w}}={\underset{\mathbf{w}}{\mathbf{T}}}^{\mathrm{T}}\left[\begin{array}{ccccc}
0.048769 & 0.045256 & -0.019229 & 0.020691 & 0.042700 \\
0.045256 & 0.043773 & -0.017865 & 0.019773 & 0.040688 \\
-0.019229 & -0.017865 & 0.015903 & -0.005882 & -0.016776 \\
0.020691 & 0.019773 & -0.005882 & 0.011111 & 0.018781 \\
0.042700 & 0.040688 & -0.016776 & 0.018781 & 0.039127
\end{array}\right] \underset{\sim}{\mathbf{w}}
$$




$$
\begin{gathered}
\underset{\sim}{\mathbf{w}}=\left[\begin{array}{l}
w_{1} \\
w_{2} \\
w_{3} \\
w_{4} \\
w_{5}
\end{array}\right] \\
\underset{\sim}{C}=\left[\begin{array}{l}
1.63342 \\
1.52597 \\
1.08618 \\
1.39108 \\
1.48821
\end{array}\right] \\
\underset{\sim}{1}=\left[\begin{array}{l}
1 \\
1 \\
1 \\
1 \\
1
\end{array}\right]
\end{gathered}
$$

\section{Resultados}

Después de resolver este problema de Programación Cuadrática la composición del portafolio

\begin{tabular}{|c|c|c|c|c|c|}
\hline \multirow[t]{2}{*}{ Portafolio } & $\begin{array}{c}\text { Resultante } \\
\text { CFE }\end{array}$ & POTOSINO & NUEVA ERA & UNIVERSAL & BUFNDÍA \\
\hline & Tarifa CFE & CFE - $6 \%$ & $\begin{array}{l}70 \text { USD / } \\
\text { MW-h }\end{array}$ & $\begin{array}{c}\text { CFE - } 16 \% \text { C/ } \\
\text { PISO }\end{array}$ & $\begin{array}{l}\text { COSTO DE } \\
\text { GENERACIÓN }\end{array}$ \\
\hline mposición & $0 \%$ & $0 \%$ & $85 \%$ & $0 \%$ & $15 \%$ \\
\hline
\end{tabular}
resultó como se muestra en la Tabla 4.

Tabla 4

Fuente: Elaboración propia con datos de CFE, Banco de México y la Reserva Federal de los Estados Unidos.

La selección sería $85 \%$ de consumo a la opción de Nueva Era, con tarifa en USD, y $15 \%$ de la opción de Comercializadora Buendía.

El costo de este portafolio es de \$1.1464 MXN por kilowatt-hora, en tarifa monómica. La varianza del portafolio esde 0.0081 (desviación estándar de 0.0900).

Es importante reconocer que la metodología considera que las opciones son infinitamente divisibles, como comentan Kumar, Mohanta y Reddy (2015), lo cual no es necesariamente aplicable a la realidad. Sin embargo, la información obtenida puede ser una guía valiosa en la toma decisiones sobre el portafolio de suministro de energía. Si bien, un vatímetro sólo ayuda a diferenciar el consumo de dos proveedores (CFE y otro más), lo que limita a utilizar 
únicamente un proveedor adicional a CFE por cada sitio, la empresa que aquí se analizó tiene varios sitios. Lo anterior significa que se podrían seleccionar los sitios adecuados para un proveedor (Nueva Era y CFE, por ejemplo) y el resto de los sitios para un segundo (Comercializadora Buendía y CFE). De esta manera se podría lograr una adaptación entre la solución obtenida a partir de esta metodología y la realidad de operación.

\section{Conclusiones}

En el presente trabajo se construyó un escenario hipotético, a partir de información actual y real, con un usuario y diversos suministradores en el mercado eléctrico mayorista de México, en el contexto de la Reforma Energética. En el caso construido, el usuario se enfrenta a una diversidad de opciones de suministro, de entre las cuales puede seleccionar para establecer contratos de suministro eléctrico.

El objetivo fue utilizar el Modelo de Markowitz, normalmente usado para construir portafolios de inversión, para validar su utilidad como herramienta de apoyo en la toma de decisiones de una empresa que, bajo el escenario de la apertura energética, tiene la posibilidad de buscar opciones diferentes de suministro de energía eléctrica.

El escenario construido incluye una variedad de suministradores con una estructura de precios asociada a la tecnología de generación y a su fuente de financiamiento. De esta manera, un suministrador cuya tecnología utiliza el gas natural tendrá una estructura de precios atada el precio del gas, con la volatilidad que el precio de éste tenga. Por otro lado, un suministrador cuya tecnología se basa en paneles solares fotovoltaicos, por ejemplo, tendrá una estructura de precios mayormente fijos, atada al costo del financiamiento que la instalación inicial requiere.

Para el caso de estudio de este trabajo, los precios históricos se construyeron con base en información real.

En el modelo de Markowitz se busca maximizar el rendimiento de un portafolio de inversión aceptando cierto nivel de riesgo. Con un enfoque diferente, en este trabajo se busca minimizar el costo de un portafolio de opciones de suministro de energía eléctrica para el usuario, aceptando cierto nivel de riesgo.

El portafolio de suministro resultante se forma de una composición de $85 \%$ de Nueva Era y $15 \%$ de Buendía. El primero, con un modelo de generación eólica que ofrece un precio estable a lo largo del tiempo, basado en un financiamiento en dólares, y el segundo con un modelo de generación con base en gas natural con un precio bajo, pero con una alta volatilidad histórica. Ésta es una solución que combina la estabilidad, o bajo riesgo, del costo asociado a la tecnología eólica y un costo bajo del gas natural, con un riesgo mayor, debido a la volatilidad de su precio.

El resultado del caso de estudio presentado fue consistente con los conceptos desarrollados 
por Markowitz en relación a que la diversificación reduce el riesgo. Es importante resaltar que las soluciones resultantes, a partir del uso del Modelo de Markowitz, constituyen una herramienta más para la toma de decisiones que, sin duda, podría robustecer los análisis y ayudar a mejorar el proceso de configurar un portafolio óptimo de suministro de energía eléctrica.

En este momento en que inicia el mercado eléctrico en México, las opciones para un usuario de energía eléctrica son limitadas, tanto por la poca oferta de suministro de energía eléctrica como por la falta de madurez en el mercado. Habría que tomar en cuenta que, en la medida que el mercado eléctrico en México vaya madurando, las opciones de suministro podrían ir cambiando, incluyendo contratos bilaterales bajo la nueva Ley y la opción de Usuarios Calificados Participantes del Mercado, para lo que el modelo propuesto seguiría siendo válido.

Una futura línea de investigación podría incluir en el modelo instrumentos financieros de cobertura. Los instrumentos de cobertura pueden disminuir el riesgo de aquellas opciones de suministro que se basan en hidrocarburos y proporcionar un portafolio con menor riesgo.

\section{Referencias}

Adams, R., \& Jamasb, T., (2016). Optimal Power Generation Portfolios with Renewables: An Application to the UK. EPRG Working Paper 1620, Cambridge, Working Papers in Economics. Disponible en: https://doi. org/10.17863/CAM.5884. Consultado:06/11/2016.

Awerbuch, S., \& Berger, M., (2003). Applying Portfolio Theory to EU Electricity Planning and Policy-Making, IEA/EET Working Paper. Febrero (03). Disponible en: http://citeseerx.ist.psu.edu/viewdoc/download?doi=10.1.1.521.6757\&rep=rep1\&type=pdf. Consultado:24/11/2016.

Awerbuch, S., Jansen, J., Bursken, L., \& Drennen,T., (2005) The Cost of Geothermal Energy in Western US Region: a portfolio-based approach, Research Gate. Disponible en: https://doi.org/10.2172/876243. Consultado: $18 / 10 / 2016$.

Awerbuch, S., (2006). Portfolio-Based Electricity Generation Planning: Policy Implications for Renewables and Energy Security. Mitigation and Adaptation Strategies for Global Change, Springer, 11: 693-710. Disponible en: https://doi.org/10.1007/s11027-006-4754-4. Consultado:30/08/2016.

Beltrán, H., (2009). Modern Portfolio Theory Applied to Electricity Generation Planning., Thesis to obtain the Master's Degree. University of Illinois at Urbana-Champaign

Cámara de Diputados del H. Congreso de la Unión, (2013), Decreto de la Reforma Energética, Secretaría de Servicios Parlamentarios, Diario Oficial, México. Disponible en: http://www.dof.gob.mx/nota_detalle.php?codigo=5327463\&fecha=20/1. Consultado:24/11/2016.

Cámara de Diputados del H. Congreso de la Unión, (2014). Ley de la Industria Eléctrica, Secretaría de Servicios Parlamentarios, Diario Oficial, México. Disponible en: http://www.diputados.gob.mx/LeyesBiblio/pdf/ LIElec_110814.pdf. Consultado: 13/05/2016.

Cucchiella, F., D’Adamo, I., \& Gastaldi, M., (2012). Modeling Optimal Investments with Portfolio Analysis in Electricity Markets. Energy Education Science and Technology Part A: Energy Science and Research, 30 (1): 673-692.

DeLaquil, P., \& Awerbuch, S., (2005). A Portfolio-Risk Analysis of Electricity Supply Options in the Commonwealth of Virginia, Climate Chesapeake Climate Action Network. December. Disponible en: http://www.ibrarian.net/navon/paper/A_Portfolio_Risk_Analysis_of_Electricity_Supply_O.pdf?paperid=4791133. Consultado: $18 / 10 / 2016$. 
De Llano Paz, F., Calvo Silvosa, A., \& Portos Garcia, M. (2012). The Problem of Determining the Energy Mix: from the Portfolio Theroy to the Reality of Energy Planning in the Spanish Case. European Research Studies, Volume XV, Issue 4, Special Issue on Energy, 3-30.

Delarue, E., De Jonghe, C., Belmans, R., \& D’haeseleer, W.D., (2009). Applying Portfolio Theory on the Electricity Sector: Installed Capacity versus Actual Electricity Generation. 33 (1). 12-23. Disponible en: https://lirias. kuleuven.be/bitstream/123456789/245206/1/portfolio_conf.pdf. Consultado: 10/11/2016.

Gökgöz, F., \& Atmaca, M.E., (2016). Portfolio Optimization Under Lower Partial Moment in Emerging Electricity Markets: Evidence from Turkey. Renewable and Sustainable Energy Reviews, 67: 437-449. Disponible en: https://doi.org/10.1016/j.rser.2016.09.029. Consultado: 06/11/2016.

Huisman, R., Mahiew, R.J., Schlichter, F., (2007) Electricity Portfolio Management: Optimal Peak / Off-Peak Allocations, ERIM Report Series Research in Management, ERS-2007-089-F\&A. Disponible en: https://doi. org/10.1016/j.rser.2016.09.029. Consultado: 06/11/2016.

Kumar, D., Mohanta, D.K., \& Reddy, J.B., (2015). Intelligent Optimization of Renewable Resource Mixes Incorporating the Effect of Fuel Risk, Fuel Cost and CO2 Emission. Frontiers in Energy, 9(1): 91-105. Disponible en: https://doi.org/10.1007/s11708-015-0345-y. Consultado 30/08/2016.

Lesser, J., Lowengrub, J., \& Yang, S., (2007). A Mean-Variance Portfolio Optimization of California's Generation Mix to 2020: Achieving California's 33 Percent Renewable Portfolio Standard Goal. Draft Consultant Report, Bates White LCC, California Energy Commission. July. CEC-300-2007-009-D. Disponible en : http://www. energy.ca.gov/2007publications/CEC-300-2007-009/CEC-300-2007-009-D.PDF. Consultado 18/10/2016.

Liu, M., \& Wu, F., (2010). Effect of Financial Hedging in Portfolio Selection in Electricity Markets. HKIE Transactions. 17 (2), 34-40. Disponible en : https://doi.org/10.1080/1023697X.2010.10668194. Consultado $10 / 11 / 2016$.

Lüttgens, D., \& Antons, D., (2013) A More Holistic Perspective on Corporate Technological Portfolio Planning: The Case of the Energy Sector, ISPIM Conference Proceedings, 1-17. Disponible en : http://search.proquest. com/openview/9172295290ab27d5edf5ae90dd6db658/1?pq-origsite=gscholar. Consultado: 02/10/2016.

Markowitz, H., (1952). Portfolio Selection. The Journal of Finance. 7 (1), 77-91. Disponible en: https://doi. org/10.1111/j.1540-6261.1952.tb01525.x . Consultado 18/10/2016.

Rodoulis, N., (2010). Evaluation of Cyprus' Electricity Generation Planning Using Mean-Variance Portfolio Theory. Cyprus Economic Policy Review. 4 (2), 25-42.

Roques, F., Hiroux, C., \& Saguan, M., (2010). Optimal Wind Power Deployment in Europe- A Portfolio Approach, Energy Policy, 38 (7), 3245-3256. Disponible en: https://doi.org/10.1016/j.enpol.2009.07.048. Consultado $18 / 10 / 2016$

Roques, F., Newbery, D.M., Nutall, W., (2008) Fuel Mix Diversification Incentives in Liberalized Electricity Markets: a Mean-Variance Portfolio Theory Approach, Energy Economics. 30: 1831-1849. Disponible en: https:// doi.org/10.1016/j.eneco.2007.11.008. Consultado 18/10/2016

Tola, M., (2015), Applying Modern Portfolio Theory to Plant Electricity Planning in Albania, European Scientific Journal, 11 (10), ISSN: 1857-7881.

Woo, C.K., Horowitz, I., Horii, B., \& Karimov, R.I., (2004) The Efficient Frontier for Spot and Forward Purchases: an Application to Electricity, Journal of the Operational Research Society, 55, 1130-1136. Disponible en: https://doi.org/10.1057/palgrave.jors.2601769. Consultado: 25/09/2016

Yan, C., (2011) The Optimization of Energy Portfolio Management (EPM): Framework and Simulation, Department of International Business of Southern New Hampshire University, Disponible en: http://academicarchive. snhu.edu/handle/10474/2294. Consultado: 30/08/2016

Ziegler, D., Schmitz, K., \& Weber, C., (2012). Optimal Electricity Generation Portfolios. The Impact of Price Spread Modelling. Computational Management Science, 9(3), 381-399. Disponible en: https://doi.org/10.1007/ s10287-012-0150-6. Consultado 02/10/2016 


\section{Anexo}

Tabla 1

Matriz de Costos de Opciones de Suministro

\begin{tabular}{|c|c|c|c|c|c|c|c|c|c|c|}
\hline \multirow{2}{*}{$\begin{array}{c}\text { Suministro } \\
\text { Tarifa }\end{array}$} & \multicolumn{2}{|c|}{ CFE } & \multicolumn{2}{|c|}{ POTOSINO } & \multicolumn{2}{|c|}{ NUEVA ERA } & \multicolumn{2}{|c|}{ UNIVERSAL } & \multicolumn{2}{|c|}{ BUENDÍA } \\
\hline & \multicolumn{2}{|c|}{ Tarifa CFE } & \multicolumn{2}{|c|}{ CFE - $6 \%$} & & $\begin{array}{l}\text { USD / } \\
\text { AW-h }\end{array}$ & & $\begin{array}{l}16 \% \mathrm{Cl} \\
50\end{array}$ & & $\begin{array}{l}\text { STO DE } \\
\text { RACIÓN }\end{array}$ \\
\hline $2011-01$ & $\$$ & 1.7124 & $\$$ & 1.6036 & $\$$ & 1.0017 & $\$$ & 1.3768 & $\$$ & 1.5582 \\
\hline $2011-02$ & $\$$ & 1.7222 & $\$$ & 1.6118 & $\$$ & 0.9887 & $\$$ & 1.3895 & $\$$ & 1.5664 \\
\hline $2011-03$ & $\$$ & 1.7531 & $\$$ & 1.6409 & $\$$ & 0.9766 & $\$$ & 1.4142 & $\$$ & 1.5871 \\
\hline $2011-04$ & $\$$ & 1.5820 & $\$$ & 1.4790 & $\$$ & 0.9076 & $\$$ & 1.2900 & $\$$ & 1.4692 \\
\hline $2011-05$ & $\$$ & 1.6820 & $\$$ & 1.5724 & 5 & 0.9107 & $\$$ & 1.3452 & $\$$ & 1.5274 \\
\hline $2011-06$ & $\$$ & 1.7381 & $\$$ & 1.6256 & $\$$ & 0.9455 & $\$$ & 1.3918 & $\$$ & 1.5824 \\
\hline $2011-07$ & $\$$ & 1.7603 & $\$$ & 1.6469 & $\$$ & 0.9444 & $\$$ & 1.4122 & $\$$ & 1.6086 \\
\hline $2011-08$ & $\$$ & 1.7585 & 5 & 1.6451 & 5 & 1.0210 & $\$$ & 1.4268 & $\$$ & 1.6209 \\
\hline $2011-09$ & $\$$ & 1.7776 & $\$$ & 1.6625 & $\$$ & 1.1112 & $\$$ & 1.4264 & $\$$ & 1.6135 \\
\hline $2011-10$ & $\$$ & 1.7540 & $\$$ & 1.6406 & $\$$ & 1.0420 & $\$$ & 1.3972 & $\$$ & 1.5774 \\
\hline $2011-11$ & $\$$ & 1.8664 & $\$$ & 1.7466 & $\$$ & 1.0936 & $\$$ & 1.4999 & $\$$ & 1.6821 \\
\hline $2011-12$ & $s$ & 2.0273 & 5 & 1.8989 & 5 & 1.1716 & 5 & 1.6587 & $\$$ & 1.8601 \\
\hline $2012-01$ & $\$$ & 1.9837 & $\$$ & 1.8573 & $\$$ & 1.0550 & $\$$ & 1.6096 & $\$$ & 1.7992 \\
\hline $2012-02$ & $\$$ & 1.9653 & $\$$ & 1.8391 & $\$$ & 1.0332 & $\$$ & 1.5992 & $\$$ & 1.7827 \\
\hline $2012-03$ & $\$$ & 1.8599 & $\$$ & 1.7408 & $\$$ & 1.0384 & $\$$ & 1.5120 & $\$$ & 1.6829 \\
\hline $2012-04$ & $\$$ & 1.6795 & $\$$ & 1.5701 & $\$$ & 1.0101 & $\$$ & 1.3797 & $\$$ & 1.5573 \\
\hline $2012-05$ & $\$$ & 1.6898 & $\$$ & 1.5797 & $\$$ & 1.1022 & $\$$ & 1.3638 & $\$$ & 1.5352 \\
\hline $2012-06$ & $\$$ & 1.7036 & $\$$ & 1.5934 & $\$$ & 1.0507 & $\$$ & 1.3787 & $\$$ & 1.5529 \\
\hline $2012-07$ & $\$$ & 1.7402 & 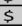 & 1.6281 & $\frac{1}{\$}$ & 1.0341 & $\$$ & 1.4115 & $\$$ & 1.5921 \\
\hline $2012-08$ & $\$$ & 1.7824 & $\$$ & 1.6674 & $\$$ & 1.0684 & $\$$ & 1.4629 & $\$$ & 1.6435 \\
\hline $2012-09$ & $\$$ & 1.6944 & $\$$ & 1.5847 & $\$$ & 1.0073 & $\$$ & 1.3749 & $\$$ & 1.5406 \\
\hline $2012-10$ & $\$$ & 1.6414 & $\$$ & 1.5353 & $\$$ & 1.0120 & $\$$ & 1.3209 & $\$$ & 1.4781 \\
\hline $2012-11$ & $\$$ & 1.7923 & $\$$ & 1.6773 & $\$$ & 1.0192 & $\$$ & 1.4547 & $\$$ & 1.6162 \\
\hline $2012-12$ & $\$$ & 1.9269 & $\$$ & 1.8049 & $\$$ & 1.0779 & $\$$ & 1.5917 & $\$$ & 1.7697 \\
\hline $2013-01$ & $\$$ & 1.8738 & $\$$ & 1.7545 & $\$$ & 1.0174 & $\$$ & 1.5331 & $\$$ & 1.7012 \\
\hline $2013-02$ & $\$$ & 1.7885 & $\$$ & 1.6738 & $\$$ & 1.0171 & $\$$ & 1.4681 & $\$$ & 1.6253 \\
\hline $2013-03$ & \$ & 1.7523 & $\$$ & 1.6401 & $\frac{1}{\$}$ & 0.9783 & $\$$ & 1.4374 & $\$$ & 1.5861 \\
\hline $2013-04$ & $\$$ & 1.6268 & $\$$ & 1.5208 & $\$$ & 0.9129 & $\$$ & 1.3507 & $\$$ & 1.5072 \\
\hline $2013-05$ & $\$$ & 1.7135 & $\$$ & 1.6018 & $\$$ & 0.9618 & $\$$ & 1.3988 & $\$$ & 1.5537 \\
\hline $2013-06$ & $\$$ & 1.7129 & $\$$ & 1.6020 & $\$$ & 1.0028 & $\$$ & 1.4002 & $\$$ & 1.5581 \\
\hline $2013-07$ & 5 & 1.6794 & $\$$ & 1.5712 & $\$$ & 0.9888 & $\$$ & 1.3742 & $\$$ & 1.5352 \\
\hline $2013-08$ & $\$$ & 1.7204 & $\$$ & 1.6094 & $\$$ & 1.0470 & $\$$ & 1.4242 & $\$$ & 1.5851 \\
\hline $2013-09$ & $\$$ & 1.6717 & $\$$ & 1.5635 & $\$$ & 1.0094 & $\$$ & 1.3677 & $\$$ & 1.5184 \\
\hline $2013-10$ & $\$$ & 1.7322 & $\$$ & 1.6201 & $\$$ & 0.9809 & $\$$ & 1.4067 & $\$$ & 1.5566 \\
\hline 2013-11 & 5 & 1.8340 & 5 & 1.7162 & 5 & 1.0088 & 5 & 1.5021 & $\$$ & 1.6514 \\
\hline $2013-12$ & $\$$ & 1.9481 & $\$$ & 1.8246 & $\$$ & 1.0560 & $\$$ & 1.6246 & $\$$ & 1.7853 \\
\hline $2014-01$ & $\$$ & 1.9041 & $\$$ & 1.7827 & $\$$ & 1.0403 & $\$$ & 1.5751 & $\$$ & 1.7251 \\
\hline $2014-02$ & $\$$ & 1.9164 & $\$$ & 1.7932 & $\$$ & 1.0203 & $\$$ & 1.5885 & $\$$ & 1.7353 \\
\hline $2014-03$ & 5 & 1.8391 & 5 & 1.7212 & $\$$ & 1.0043 & $\$$ & 1.5211 & 5 & 1.6611 \\
\hline $2014-04$ & $\$$ & 1.6874 & $\$$ & 1.5774 & $\$$ & 0.9709 & $\$$ & 1.4119 & $\$$ & 1.5590 \\
\hline $2014-05$ & $\$$ & 1.6542 & $\$$ & 1.5464 & $\$$ & 0.9489 & $\$$ & 1.3624 & $\$$ & 1.5002 \\
\hline $2014-06$ & $\$$ & 1.6817 & $\$$ & 1.5728 & $\$$ & 0.9775 & $\$$ & 1.3880 & $\$$ & 1.5285 \\
\hline $2014-07$ & $\$$ & 1.6943 & $\$$ & 1.5851 & $\$$ & 0.9943 & $\$$ & 1.4011 & $\$$ & 1.5459 \\
\hline $2014-08$ & $\$$ & 1.7605 & $\$$ & 1.6468 & $\$$ & 1.0056 & $\$$ & 1.4724 & $\$$ & 1.6185 \\
\hline $2014-09$ & $\$$ & 1.7122 & $\$$ & 1.6014 & $\$$ & 1.0060 & $\$$ & 1.4157 & $\$$ & 1.5532 \\
\hline $2014-10$ & $\$$ & 1.6488 & $\$$ & 1.5422 & $\$$ & 0.9925 & $\$$ & 1.3530 & $\$$ & 1.4826 \\
\hline $2014-11$ & $\$$ & 1.7082 & $\$$ & 1.5986 & $\$$ & 1.0338 & $\$$ & 1.4137 & $\$$ & 1.5365 \\
\hline $2014-12$ & $\$$ & 1.7798 & $\$$ & 1.6673 & $\$$ & 1.1434 & $\$$ & 1.5027 & $\$$ & 1.6363 \\
\hline $2015-01$ & $\$$ & 1.6591 & $\$$ & 1.5537 & $\$$ & 1.1216 & $\$$ & 1.3844 & $\$$ & 1.5062 \\
\hline $2015-02$ & $\$$ & 1.5848 & $\$$ & 1.4833 & $\$$ & 1.1144 & $\$$ & 1.3250 & $\$$ & 1.4441 \\
\hline $2015-03$ & $\$$ & 1.4902 & $\$$ & 1.3951 & 5 & 1.1298 & $\$$ & 1.2963 & $\$$ & 1.3500 \\
\hline $2015-04$ & $\$$ & 1.4961 & $\$$ & 1.2256 & $\$$ & 1.1053 & $\$$ & 1.2511 & $\$$ & 1.2278 \\
\hline $2015-05$ & $\$$ & 1.3193 & $\$$ & 1.2022 & $\$$ & 1.0989 & $\$$ & 1.2305 & $\$$ & 1.1764 \\
\hline $2015-06$ & $\$$ & 1.2820 & $\$$ & 1.1946 & $\$$ & 1.1460 & $\$$ & 1.2518 & $\$$ & 1.1704 \\
\hline $2015-07$ & $\$$ & 1.2734 & $\$$ & 1.1867 & $\$$ & 1.1781 & $\$$ & 1.2547 & $\$$ & 1.1657 \\
\hline $2015-08$ & $\$$ & 1.2667 & $\$$ & 1.2333 & $\$$ & 1.2605 & $\$$ & 1.2856 & $\$$ & 1.2364 \\
\hline $2015-09$ & $\$$ & 1.3107 & $\$$ & 1.2735 & $\$$ & 1.2285 & $\$$ & 1.2594 & $\$$ & 1.2411 \\
\hline $2015-10$ & $\$$ & 1.3534 & $\$$ & 1.2162 & $\$$ & 1.1851 & $\$$ & 1.2502 & $\$$ & 1.1687 \\
\hline $2015-11$ & $\$$ & 1.3127 & $\$$ & 1.2290 & $\$$ & 1.2092 & $\$$ & 1.2862 & $\$$ & 1.1901 \\
\hline $2015-12$ & $\$$ & 1.3206 & $\$$ & 1.2386 & $\$$ & 1.3157 & $\$$ & 1.3493 & $\$$ & 1.2355 \\
\hline $2016-01$ & $\$$ & 1.2871 & $\$$ & 1.2062 & $\$$ & 1.3344 & $\$$ & 1.3106 & $\$$ & 1.1834 \\
\hline $2016-02$ & $\$$ & 1.3405 & 5 & 1.2551 & $\$$ & 1.3167 & $\$$ & 1.3055 & $\$$ & 1.2305 \\
\hline $2016-03$ & $\$$ & 1.2296 & $\$$ & 1.1520 & $\$$ & 1.2451 & $\$$ & 1.2953 & $\$$ & 1.1224 \\
\hline $2016-04$ & $\$$ & 1.2440 & $\$$ & 1.1631 & $\$$ & 1.2139 & $\$$ & 1.2592 & $\$$ & 1.1730 \\
\hline $2016-05$ & $\$$ & 1.1563 & $\$$ & 1.0814 & $\$$ & 1.2998 & $\$$ & 1.2387 & $\$$ & 1.0646 \\
\hline $2016-06$ & $\$$ & 1.2227 & $\$$ & 1.1441 & $\$$ & 1.3337 & $\$$ & 1.2609 & $\$$ & 1.1377 \\
\hline $2016-07$ & $\$$ & 1.3050 & $\$$ & 1.2213 & $\$$ & 1.3500 & $\$$ & 1.2648 & $\$$ & 1.2125 \\
\hline $2016-08$ & $\$$ & 1.3932 & $\$$ & 1.3039 & $\$$ & 1.3825 & $\$$ & 1.3004 & $\$$ & 1.3018 \\
\hline $2016-09$ & 5 & 1.4342 & 5 & 1.3415 & $\$$ & 1.3867 & $\$$ & 1.2714 & $\$$ & 1.3074 \\
\hline $2016-10$ & $\$$ & 1.4248 & $\$$ & 1.3331 & $\$$ & 1.3349 & $\$$ & 1.2622 & $\$$ & 1.2793 \\
\hline
\end{tabular}

Fuente: Elaboración propia con datos de CFE, Banco de México y la Reserva Federal de los Estados Unidos. 